\title{
Convection of Radiating Gas in a Vertical Channel through Porous Media
}

\author{
Mrinal Jana, Sovan Lal Maji, Sanatan Das, Rabindranath Jana \\ Department of Applied Mathematics, Vidyasagar University, Midnapore, India \\ E-mail: jana261171@yahoo.co.in \\ Received September 12, 2011; revised October 10, 2011; accepted October 31, 2011
}

\begin{abstract}
The free convection flow of radiating gas between two vertical thermally conducting walls through porous medium in the presence of a uniform gravitational field has been studied. Closed form solutions for the velocity and temperature have been obtained in the optically thin limit case when the wall temperatures are varying linearly with the vertical distance. It is observed that the fluid velocity increases and the temperature difference between the walls and the fluid decreases with an increase in the radiation parameter. It is also observed that both the fluid velocity and temperature in the flow field increase with an increase in the porosity parameter. It is found that the fluid velocity decreases while the temperature increases with an increase in the thermal conductance of the walls. Further, it is found that radiation causes to decrease the rate of heat transfer to the fluid, thereby reducing the effect of natural convection.
\end{abstract}

Keywords: Radiation, Porous Medium, Heat Transfer and Thermal Conductance

\section{Introduction}

In recent years, free convection flow of viscous fluids through porous medium has attracted the attention of a number of researchers in view of its wide application to geophysics, astrophysics, meteorology, aerodynamics, boundary layer control and so on. In addition, convective flow through a porous medium has the application in the field of chemical engineering for filtration and purification processes. In petroleum technology, to study the movement of natural gas oil and water through oil channels/reservoirs and in the field of agriculture engineering to study the underground resources, the channel flows through porous medium have numerous engineering and geophysical applications. However, these studies are confined to normal temperatures of the surrounding medium. If the temperature of the surrounding fluid is rather high, radiation effects play an important role and this situation does exist in space technology. In such cases, one has to take into account the effects of radiation and free convection. Channels are frequently used in various applications in designing ventilating and heating of buildings, cooling electronic components, drying several types of agriculture products grain and food, and packed bed thermal storage. Convective flows in channels driven by temperature differences at bounding walls have been studied and reported extensively in literature. Radiative convective flows are frequently encountered in many scientific and environmental processes such as astrophysical flows, water evaporation from open reservoirs, heating and cooling of chambers and solar power technology. Several researchers have investigated convective flow in porous medium such as Nield and Bejan [1], Sparrow and Cess [2], Burmeister [3], Bejan [4], Kaviany [5] and Vafai [6]. Raptis $[7,8]$ has studied the effect of radiation on free convection flow through a porous medium. The natural convection cooling of vertical rectangular channels in air considering radiation and wall conduction has been studied by Hall et al. [9]. Al-Nimr and Haddad [10] have described the fully developed free convection in open-ended vertical channels partially filled with porous material. Thermal dispersion-radiation effects on non-Darcy natural convection in a fluid saturated porous medium have been investigated by Mohammadein and El-Amin [11]. The effect of wall conductances on free convection between asymmetrically heated vertical plates has been studied by Kim et al. [12]. Greif et al. [13] have made an analysis on the laminar convection of a radiating gas in a vertical channel. Effect of wall conductances on convective magnetohydrodynamic channel flow has been investigated by Yu and Yang [14]. Gupta and Gupta [16] have studied the radiation effect on hydromagnetic convection 
in a vertical channel. Datta and Jana [15] have studied the effect of wall conductances on hydromagnetic convection of a radiation gas in a vertical channel. Makinde and Mhone [17] investigated the effect of thermal radiation on MHD oscillatory flow in a channel filled with saturated porous medium and non-uniform wall temperatures. Narahari [18] has investiagted the effects of thermal radiation and free convection currents on the unsteady Couette flow between two vertical parallel plates with constant heat flux at one boundary.

In this paper, we have studied the fully developed free convection flow of radiating gas between two vertical thermally conducting walls embedded in porous medium. The governing equations are solved analytically. The effects of the permeability of the porous medium and the influences of radiation parameter and thermal wall conductances on velocity filed and temperature distribution are investigated and analyzed with the help of their graphical representations. It is observed that the fluid velocity $u_{1}(\eta)$ increases whereas the temperature distribution $\theta(\eta)$ decreases with an increase in the radiation parameter $F$. It is also observed that both the fluid velocity $u_{1}(\eta)$ and temperature $\theta(\eta)$ in the flow field increase with an increase in the porosity parameter $\sigma$. It is found that the fluid velocity decreases while the temperature increases with an increase in the thermal conductance $\psi$. Further, it is found that radiation causes to decrease the rate of heat transport to the fluid thereby reducing the effect of natural convection. The rate of flow increases with an increase in either radiation parameter $F$ or Rayleigh number

\section{Formulation of the Problem and Its Solutions}

Consider a fully developed flow of a viscous incompressible fluid flow in a vertical channel embedded in porous medium. The distance between the channel walls is $2 L$. Employ a Cartesian coordinates system with $z-$ axis vertically upwards along the direction of flow and $y$-axis perpendicular to it. The origin of the axes is such that the channel walls are at positions $y=-L$ and $y=L$ (see Figure 1).

For the fully developed laminar flow in porous medium, the velocity and the temperature field have only a vertical component and all of the physical variables except temperature and pressure are functions of $y$. The temperature inside the fluid can be written as

$$
T=T^{*}(y)+N z,
$$

where $N$ is the vertical temperature gradient.

On the use of (1), the momentum and energy equations are simplified to the following form

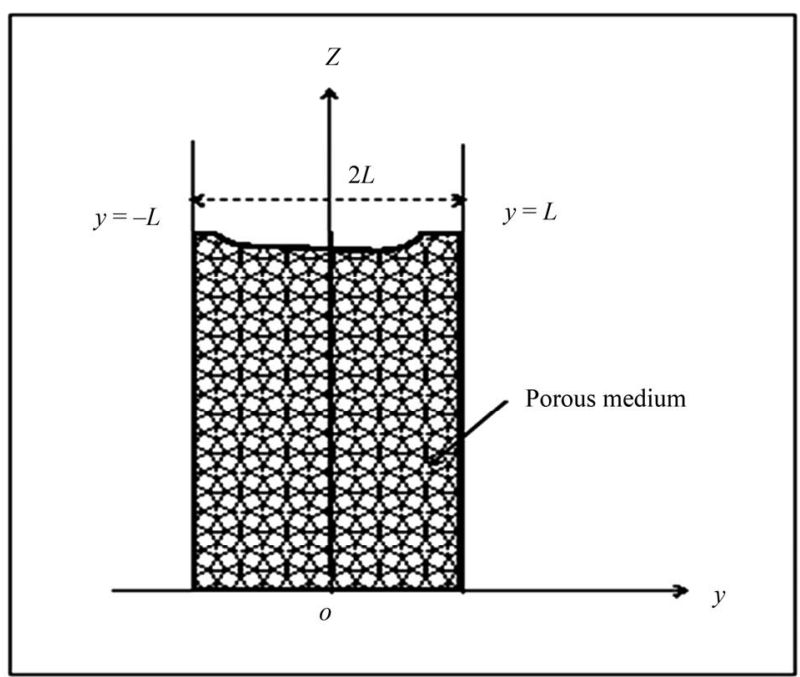

Figure 1. Geometry of the problem.

$$
\begin{gathered}
v \frac{\mathrm{d}^{2} u}{\mathrm{~d} y^{2}}-\frac{v}{k^{\prime}} u+g \beta\left(\theta^{*}+N z\right)-\frac{1}{\rho} \frac{\partial p}{\partial z}=0, \\
-\frac{1}{\rho} \frac{\partial p}{\partial z}=0, \\
N u=\alpha \frac{\mathrm{d}^{2} \theta^{*}}{\mathrm{~d} y^{2}}-\frac{1}{\rho c_{p}} \frac{\partial q_{r}}{\partial y},
\end{gathered}
$$

where $\theta^{*}=T-T_{w_{0}}, v$ is the kinematic coefficient of fluid viscosity, $g$ the acceleration due to gravity, $k$ permeability of the porous medium and $\alpha$ the thermal conductivity.

In the optically thin limit, the fluid does not absorb its own emitted radiation. This means that there is no selfabsorption but the fluid does absorb radiation emitted by the boundaries. Cogley et al. [19] showed that in the optically thin limit for a non-grey gas near equilibrium, the following relation holds

$$
\frac{\partial q_{r}}{\partial y}=4\left(T-T_{w}\right) \int_{0}^{\infty} K_{\lambda_{w}}\left(\frac{\partial e_{\lambda h}}{\partial T}\right)_{w} \mathrm{~d} \lambda,
$$

where $K_{\lambda}$, is the absorption coefficient, $e_{\lambda h}$ is the Planck function and the subscript $w$ refers to values at the wall. Further simplifications can be made concerning the spectral properties of radiating gases ([20]) but are not necessary for our investigation.

On the use of (5), Equation (4) becomes

$$
N u=\alpha \frac{\mathrm{d}^{2} \theta^{*}}{\mathrm{~d} y^{2}}-c \theta^{*}
$$

where

$$
c=\frac{4}{\rho c_{p}} \int_{0}^{\infty} K_{\lambda_{0}}\left(\frac{\partial e_{\lambda h}}{\partial T}\right)_{0} \mathrm{~d} \lambda .
$$


Subscript "0" indicates that all quantities have been evaluated at the entrance temperature $T_{w 0}$ which is the temperature of the wall at $z=0$.

Integrating Equation (3) we get

$$
p=f(z) \text {. }
$$

On use of (8), Equation (2) becomes

$$
v \frac{\mathrm{d}^{2} u}{\mathrm{~d} y^{2}}+g \beta \theta^{*}-\frac{v}{k^{\prime}} u=-c_{1},
$$

where

$$
c_{1}=-\left[\frac{1}{\rho} \frac{\partial f}{\partial z}-g \beta N z\right] .
$$

Introducing the non-dimensional variables

$$
\eta=\frac{y}{L}, u_{1}=\frac{u L}{\alpha c_{2}}, \theta=-\frac{\theta^{*}}{N L c_{2}}, c_{2}=\frac{c_{1} L^{3}}{v \alpha}
$$

and on using (11), Equations (9) and (6) become

$$
\begin{aligned}
& \frac{\mathrm{d}^{2} u_{1}}{\mathrm{~d} \eta^{2}}-R a \theta-\frac{1}{\sigma} u_{1}=-1, \\
& \frac{\mathrm{d}^{2} \theta}{\mathrm{d} \eta^{2}}-F \theta=-u_{1},
\end{aligned}
$$

where $\sigma=\frac{k^{\prime}}{L^{2}}$ is the porosity parameter, $R a=\frac{g \beta N L^{4}}{v \alpha}$ is the Rayleigh number and $F=\frac{c L^{2}}{\alpha}$ is the radiation parameter.

The dimensionless velocity and the temperature boundary conditions are

$$
\begin{gathered}
u_{1}=0 \text { at } \eta= \pm 1, \\
\frac{\mathrm{d} \theta}{\mathrm{d} \eta} \pm \frac{\theta}{\psi}=0 \text { at } \eta= \pm 1
\end{gathered}
$$

where $\psi$ is the thermal conductance ratio.

Eliminating $u_{1}$ from (12) and (13), we obtain

$$
\frac{\mathrm{d}^{4} \theta}{\mathrm{d} \eta^{4}}-\left(\frac{1}{\sigma}+F\right) \frac{\mathrm{d}^{2} \theta}{\mathrm{d} \eta^{2}}+\left(R a+\frac{F}{\sigma}\right) \theta=1 .
$$

The solution of $\theta(\eta)$ satisfying the boundary conditions (14) is easily obtained. Achieving $\theta(\eta)$, one can determine $u_{1}(\eta)$ from (12) using the boundary conditions (14).

The solutions for $\theta(\eta)$ and $u_{1}(\eta)$ subject to the boundary conditions (14) are

$$
\begin{aligned}
& \theta(\eta)=a_{4} {\left[1-a_{2} \cosh m_{2} \eta-a_{3}\left\{\cosh m_{1} \eta-a_{1} \cosh m_{2} \eta\right\}\right], } \\
& u_{1}(\eta)=a_{4}\left[F-a_{2}\left(F-m_{2}^{2}\right) \cosh m_{2} \eta\right. \\
&\left.\quad-a_{3}\left\{\left(F-m_{1}^{2}\right) \cosh m_{1} \eta-a_{1}\left(F-m_{2}^{2}\right) \cosh m_{2} \eta\right\}\right]
\end{aligned}
$$

where

$$
\begin{aligned}
& a_{1}=\frac{\psi m_{1} \sinh m_{1}+\cosh m_{1}}{\psi m_{2} \sinh m_{2}+\cosh m_{2}} \\
& a_{2}=\frac{1}{\psi m_{2} \sinh m_{2}+\cosh m_{2}}, \\
& a_{3}=\frac{F-a_{2}\left(F-m_{2}^{2}\right) \cosh m_{2}}{\left(F-m_{1}^{2}\right) \cosh m_{1}-a_{1}\left(F-m_{2}^{2}\right) \cosh m_{2}}, \\
& a_{4}=\frac{1}{R a+F / \sigma}, \\
& m_{1}^{2}=\frac{1}{2}\left[\left(\frac{1}{\sigma}+F\right)+\left\{\left(\frac{1}{\sigma}-F\right)^{2}-4 R a\right\}^{1 / 2}\right], \\
& m_{2}^{2}=\frac{1}{2}\left[\left(\frac{1}{\sigma}+F\right)-\left\{\left(\frac{1}{\sigma}-F\right)^{2}-4 R a\right\}^{1 / 2}\right]
\end{aligned}
$$

It is observed from the Equations (16) and (17) that the velocity and temperature depend on the parameters $\sigma, F, R a$ and $\psi$.

Case-I: Constant wall temperature $(\psi=0)$.

The temperature distribution $\theta(\eta)$ and velocity $u_{1}(\eta)$ for constant wall temperature are given by

$$
\begin{aligned}
\theta(\eta)=\frac{1}{\left(R a+\frac{F}{\sigma}\right)}\left[1-\frac{m_{2}^{2}}{m_{2}^{2}-m_{1}^{2}} \frac{\cosh m_{1} \eta}{\cosh m_{1}}\right. \\
\left.+\frac{m_{1}^{2}}{m_{2}^{2}-m_{1}^{2}} \frac{\cosh m_{2} \eta}{\cosh m_{2}}\right], \\
\begin{aligned}
u_{1}(\eta)=\frac{1}{\left(R a+\frac{F}{\sigma}\right)}\left[F-\frac{\left(F-m_{1}^{2}\right) m_{2}^{2}}{m_{2}^{2}-m_{1}^{2}} \frac{\cosh m_{1} \eta}{\cosh m_{1}}\right. \\
\left.+\frac{\left(F-m_{2}^{2}\right) m_{1}^{2}}{m_{2}^{2}-m_{1}^{2}} \frac{\cosh m_{2} \eta}{\cosh m_{2}}\right],
\end{aligned}
\end{aligned}
$$

where $m_{1}$ and $m_{2}$ are given by (19).

Case-II: Thermally insulated walls $(\psi=\infty)$.

The temperature distribution $\theta(\eta)$ and velocity $u_{1}(\eta)$ for thermally insulated walls are given by

$$
\theta(\eta)=\frac{1}{\left(R a+\frac{F}{\sigma}\right)}\left[\begin{array}{c}
F\left(m_{2} \sinh m_{2} \cosh m_{1} \eta\right. \\
1-\frac{\left.-m_{1} \sinh m_{1} \cosh m_{2} \eta\right)}{m_{2}\left(F-m_{1}^{2}\right) \sinh m_{2} \cosh m_{1}} \\
-m_{1}\left(F-m_{2}^{2}\right) \sinh m_{1} \cosh m_{2}
\end{array}\right]
$$




$$
u_{1}(\eta)=\frac{F}{\left(R a+\frac{F}{\sigma}\right)}\left[\begin{array}{r}
m_{2}\left(F-m_{1}^{2}\right) \sinh m_{2} \cosh m_{1} \eta \\
1-\frac{-m_{1}\left(F-m_{2}^{2}\right) \sinh m_{1} \cosh m_{2} \eta}{m_{2}\left(F-m_{1}^{2}\right) \sinh m_{2} \cosh m_{1}} \\
-m_{1}\left(F-m_{2}^{2}\right) \sinh m_{1} \cosh m_{2}
\end{array}\right] \text {, }
$$

where $m_{1}$ and $m_{2}$ are given by (19).

\section{Results and Discussion}

To study the effects of radiation and porosity of the porous medium on the velocity field $u_{1}$ and temperature distribution $\theta$, we have presented the non-dimensional velocity $u_{1}$ and the temperature $\theta$ against $\eta$ for various values of radiation parameter $F$, Rayleigh number $R a$, porosity parameter $\sigma$ and the thermal conductance parameter $\psi$ in Figures 2-9. It is observed from Figure 2 that the velocity $u_{1}(\eta)$ increases with an increase in radiation parameter $F$. Increasing the radiation parameter $F$ produces significant increase in the thermal condition of the fluid. This increase in the fluid temperature induces more flow causing the velocity of the fluid there to increase. Figure 3 shows that the velocity at any point in the flow region decreases with an increase in Rayleigh number $R a$. The Rayleigh number is viewed as the ratio of buoyancy forces and the product of thermal and momentum diffusivities. Increasing value of Rayleigh number opposes the natural convection and hence the fluid velocity decreases. It is observed from Figure 4 that the velocity $u_{1}$ increases with an increase in porosity parameter $\sigma$. Porosity is a measure of the void (or empty) spaces in a porous medium and is a fraction of the volume of voids over the total volume. Porosity influences the convection flow and so the fluid velocity increases. It is seen from Figure $\mathbf{5}$ that the velocity decreases with an

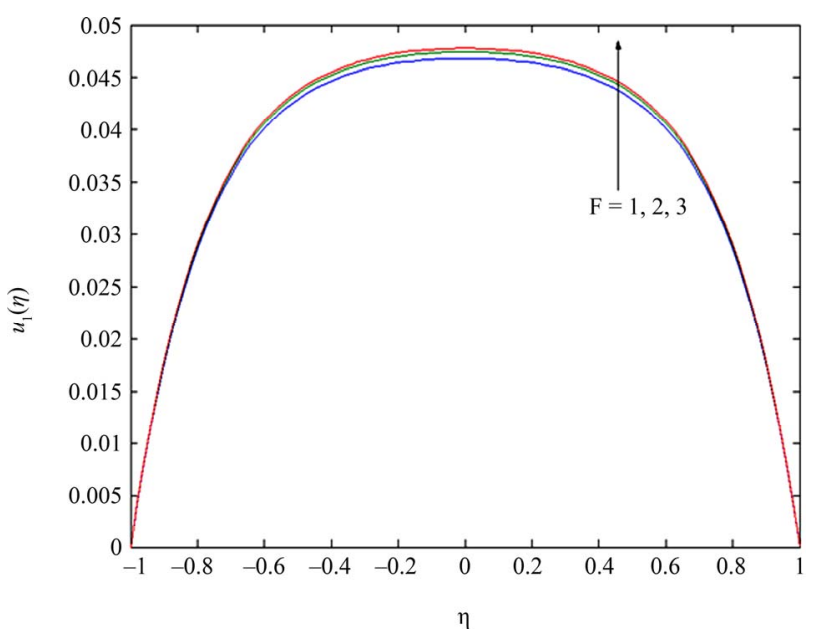

Figure 2. Variations of $u_{1}(\eta)$ for $R a=2, \psi=0.5$ and $\sigma=0.1$.

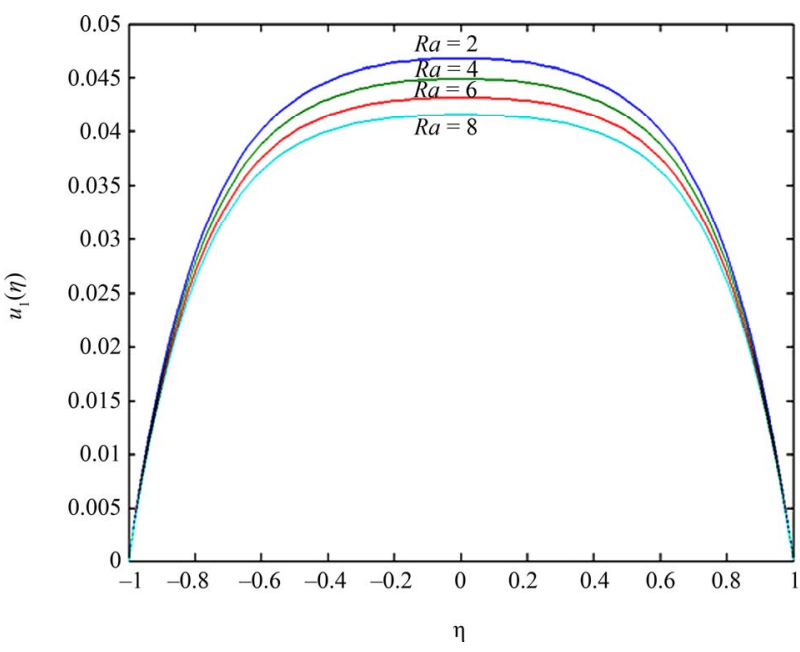

Figure 3. Variations of $u_{1}(\eta)$ for $F=1, \psi=0.5$ and $\sigma=0.1$.

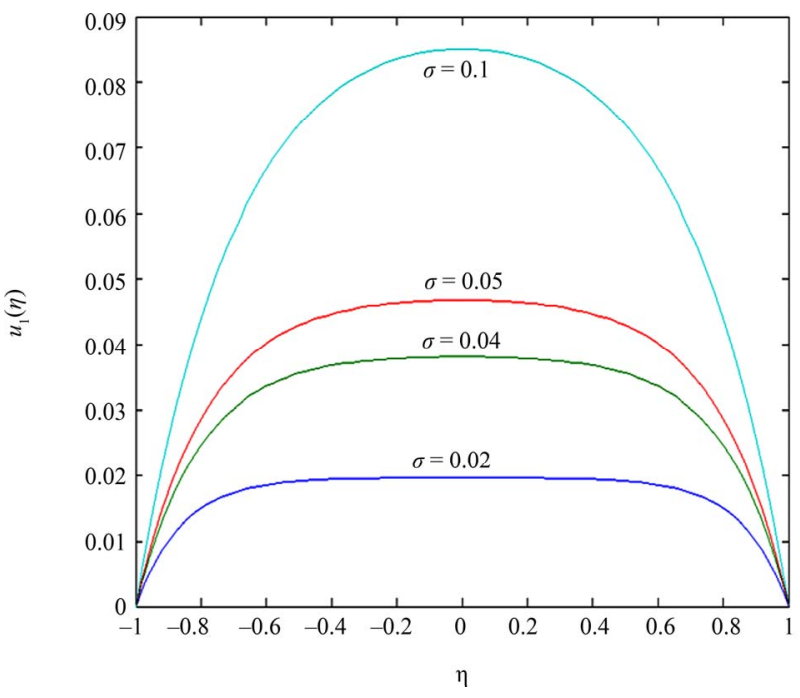

Figure 4. Variations of $u_{1}(\eta)$ for $F=1, \psi=0.5$ and $R a=1$.

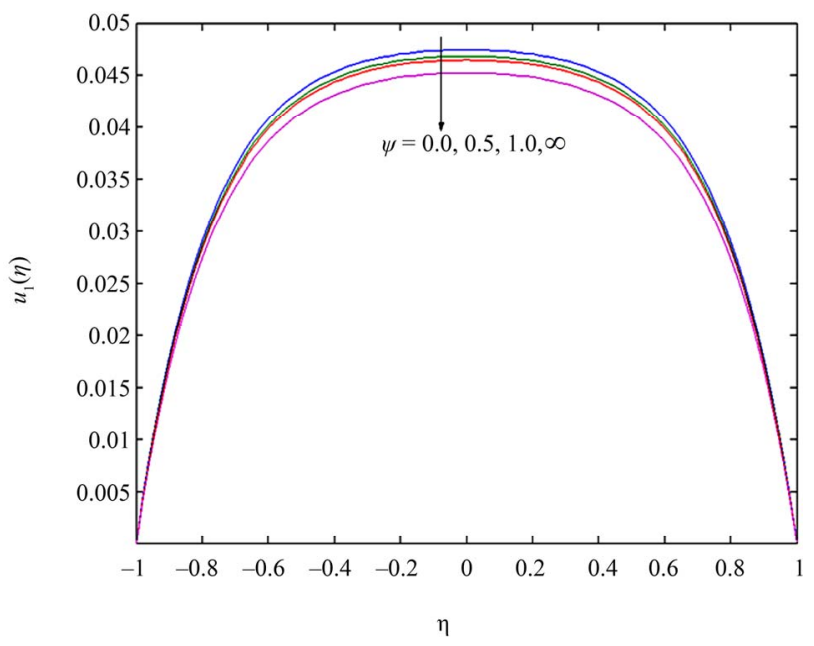

Figure 5. Variations of $u_{1}(\eta)$ for $F=1, \sigma=0.1$ and $R a=1$. 


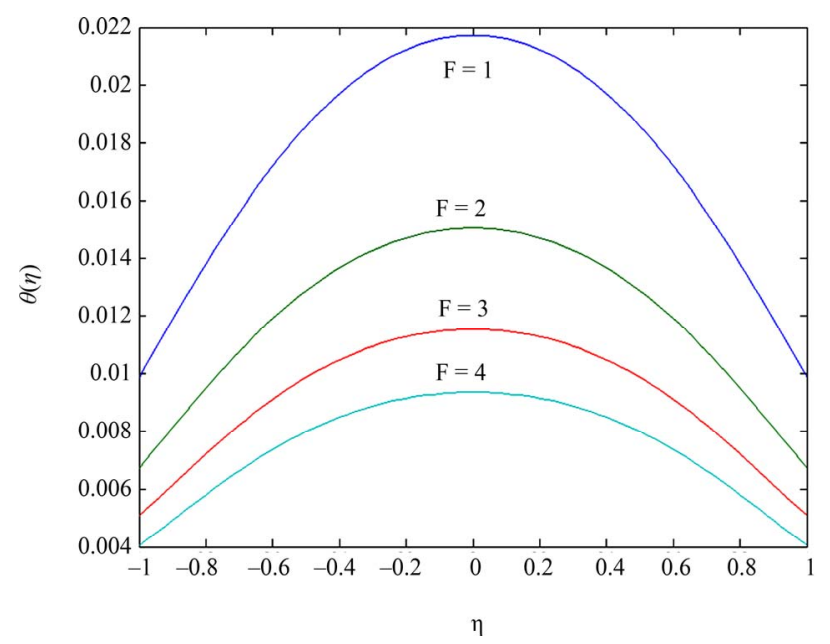

Figure 6. Variations of $\theta(\eta)$ for $\psi=0.5, \sigma=0.1$ and $R a=1$.

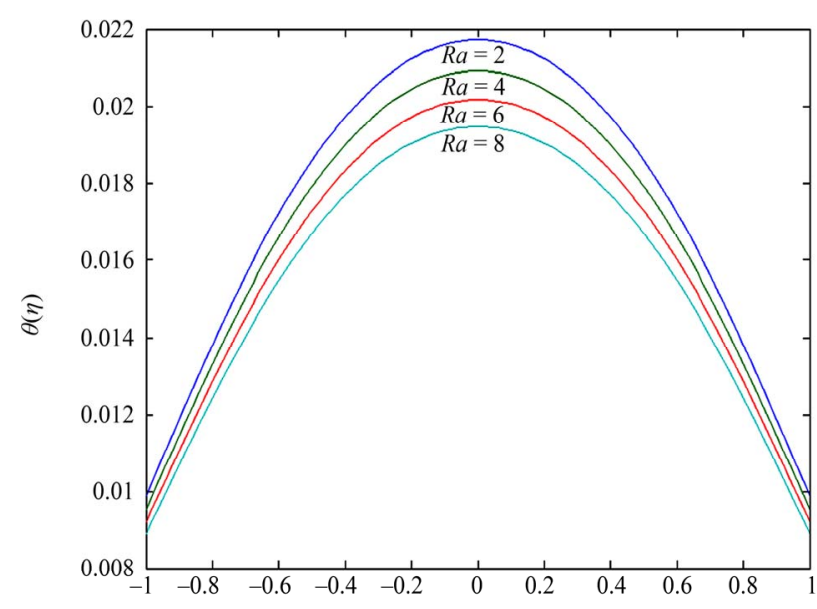

$\eta$

Figure 7. Variations of $\theta(\eta)$ for $F=1, \sigma=0.1$ and $\psi=0.5$.

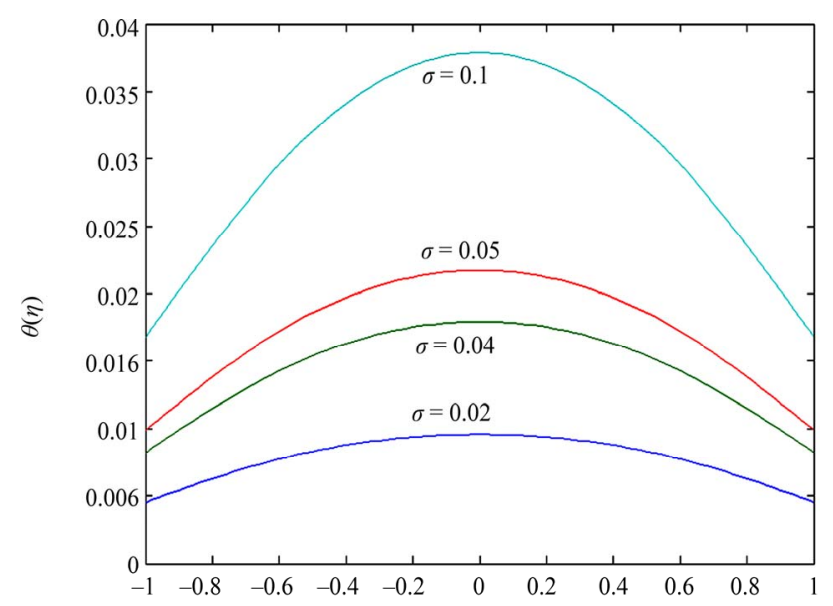

$\eta$

Figure 8. Variations of $\theta(\eta)$ for $F=1, \psi=0.5$ and $R a=1$.

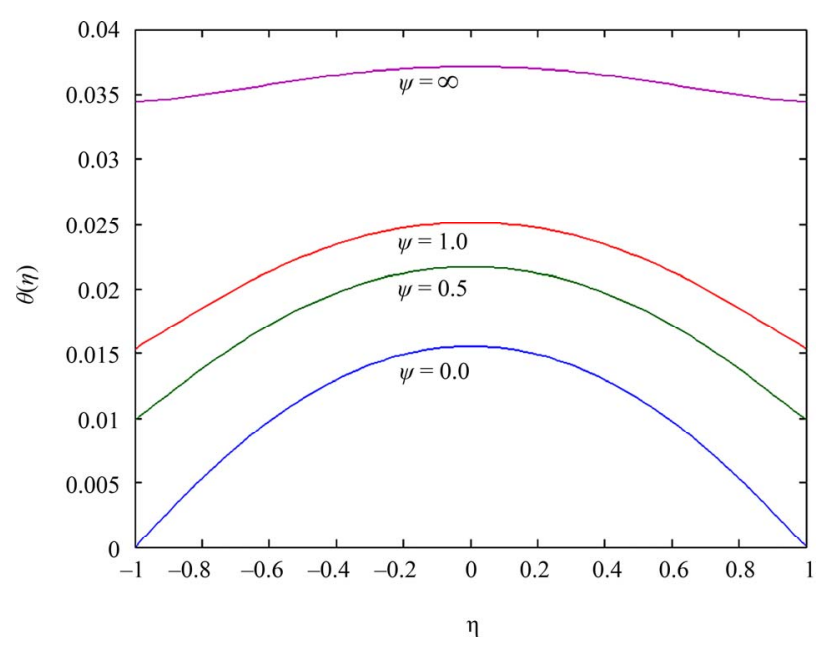

Figure 9. Variations of $\theta(\eta)$ for $F=1, \sigma=0.1$ and $R a=1$.

increase in thermal conductance parameter $\psi$. Figures 6 and 7 reveal that the temperature $\theta$ decreases with an increase in either radiation parameter $F$ or Rayleigh number $R a$. Radiation tends to increase the rate of heat transport to the fluid. Thus the effect of radiation reduces the influence of natural convection by causing a reduction in the temperature difference between the fluid and the channel walls. The increase in radiation parameter $F$ means the release of heat energy from the flow region and so the fluid temperature significantly decreases. It is found from Figures 8 and 9 that the temperature $\theta$ increases with an increase in either porosity parameter $\sigma$ or thermal conductance parameter $\psi$.

The non-dimensional shear stress at the right wall $(\eta=1)$ of the channel is given by

$$
\begin{aligned}
\tau=-a_{4}[ & a_{3}\left(F-m_{1}^{2}\right) m_{1} \sinh m_{1} \\
& \left.+\left(a_{2}-a_{1} a_{3}\right)\left(F-m_{2}^{2}\right) m_{2} \sinh m_{2}\right] .
\end{aligned}
$$

Numerical values of non-dimensional shear stress $\tau$ at the right wall $(\eta=1)$ of the channel are plotted against $F$ for different values of $R a, \sigma$ and $\psi$ in Figures 10-12. It is observed from Figure 10 that for fixed values of $F$ the magnitude of the shear stress $\tau$ at the right wall decreases with an increase in Rayleigh number $R a$. Figure 11 reveals that the magnitude of $\tau$ increases with an increase in porosity parameter $\sigma$. On the other hand, it is seen from Figure 12 that for fixed values of $F$, the magnitude of $\tau$ decreases with an decrease in $\psi$.

The rate of heat transfer across the channel's wall is given as

$\left.-\frac{d \theta}{d \eta}\right]_{\eta=1}=a_{4}\left[a_{3} m_{1} \sinh m_{1}+\left(a_{2}-a_{1} a_{3}\right) m_{2} \sinh m_{2}\right]$. 


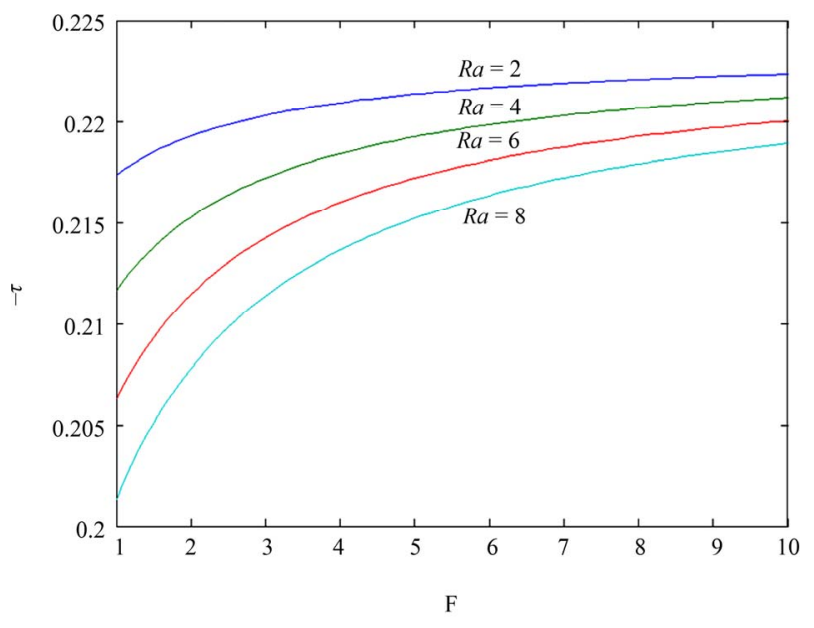

Figure 10. Variations of $-\tau$ against $F$ for $\psi=0.5$ and $\sigma=0.1$.

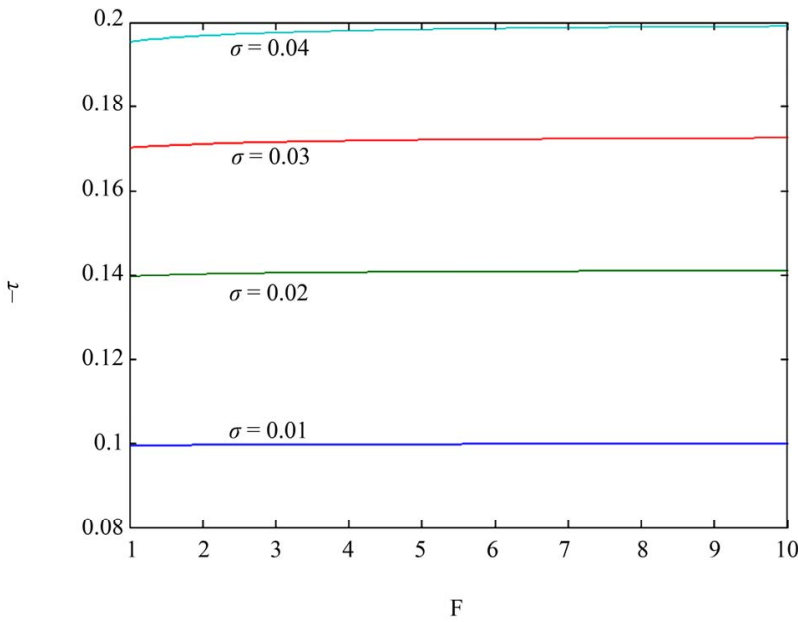

Figure 11. Variations of $-\tau$ against $F$ for $\psi=0.5$ and $R a=2$.

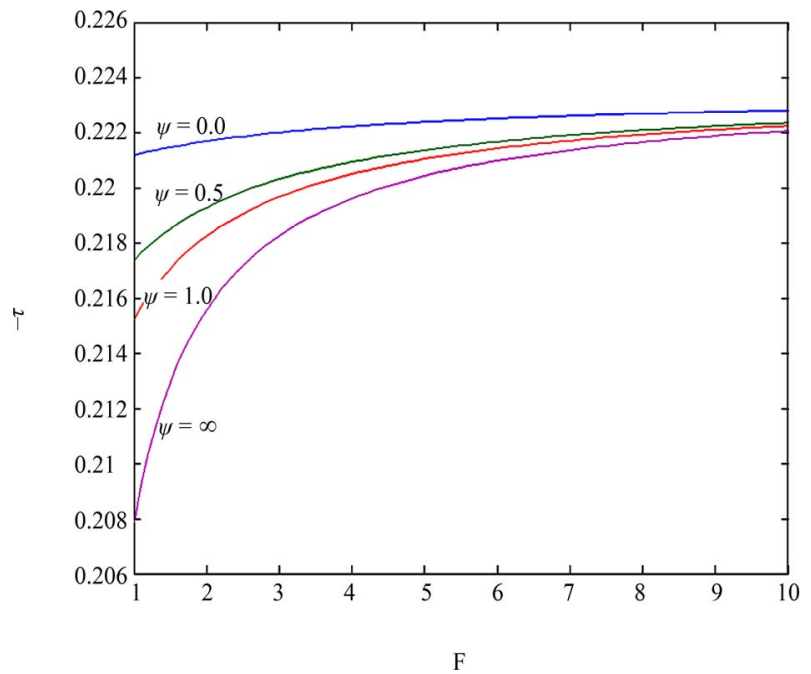

Figure 12. Variations of shear stress $-\tau$ against $F$ for $R a=2$ and $\sigma=0.1$.
Numerical values of the rate of heat transfer $-\theta^{\prime}(1)$ are shown graphically against $F$ for different values of $R a, \sigma$ and $\psi$ in Figures 13-15. It is observed from Figure 13 that for fixed values of $F$, the rate of heat transfer $-\theta^{\prime}(1)$ decreases with an increase in Rayleigh number $R a$. Figure 14 reveals that the $-\theta^{\prime}(1)$ increases with an increase in porosity parameter $\sigma$. On the other hand, it is seen from Figure 15 that for fixed values of $F$, $-\theta^{\prime}(1)$ decreases with an decrease in $\psi$.

The non-dimensional flow rate is given by

$$
\begin{aligned}
W= & \int_{-1}^{1} u_{1}(\eta) \mathrm{d} \eta \\
=2 a_{4}[ & {\left[F-a_{3}\left(F-m_{1}^{2}\right) \frac{\sinh m_{1}}{m_{1}}\right.} \\
& \left.\quad-\left(a_{2}-a_{1} a_{3}\right)\left(F-m_{2}^{2}\right) \frac{\sinh m_{2}}{m_{2}}\right] .
\end{aligned}
$$

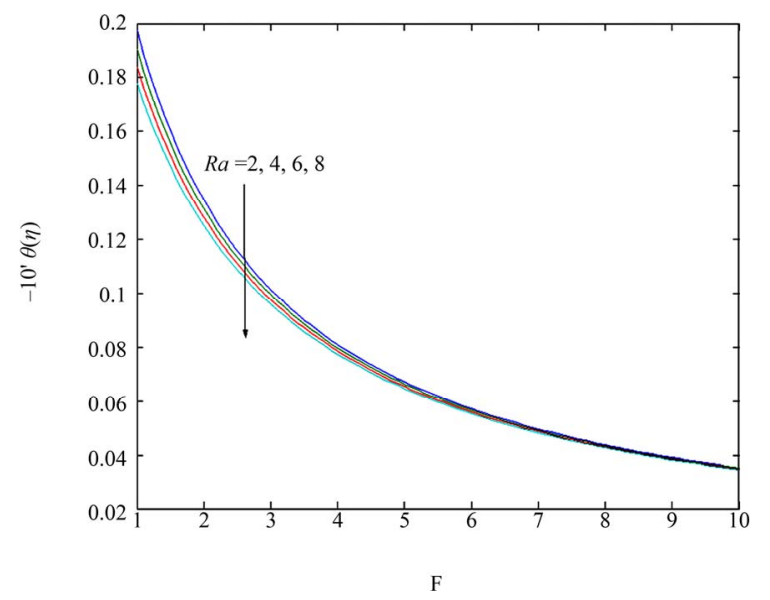

Figure 13. Variations of $-\theta^{\prime}(1)$ against $F$ for $\psi=0.5$ and $\sigma=$ 0.1.

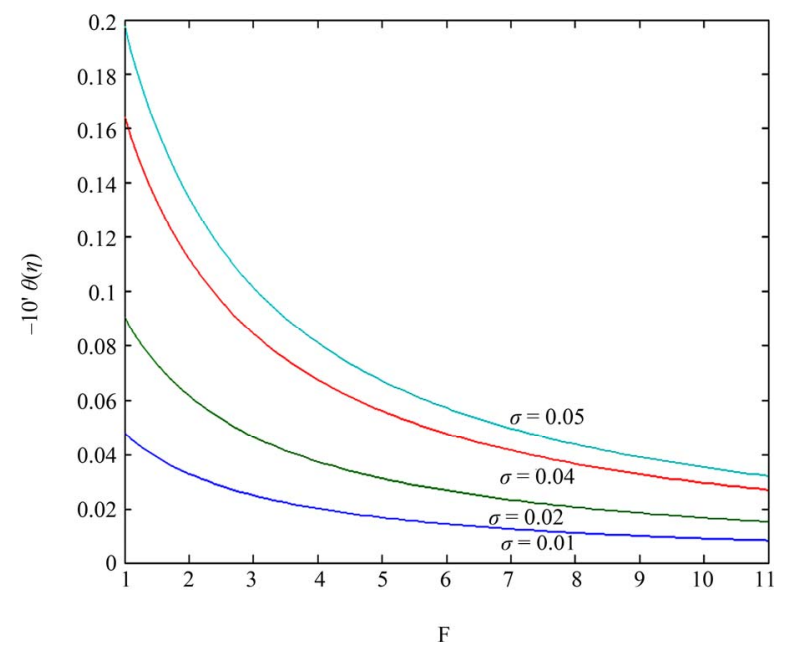

Figure 14. Variations of $-\theta^{\prime}(1)$ against $F$ for $\psi=0.5$ and $R a=$ 2. 


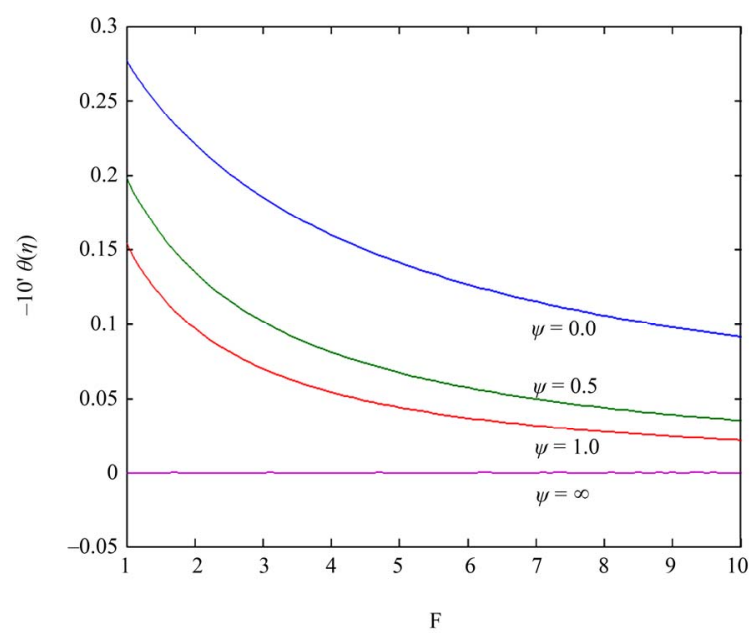

Figure 15. Variations of $-\theta^{\prime}(1)$ against $F$ for $\sigma=0.1$ and $R a$ $=2$.

The non-dimensional flow rate, $W$ has been plotted against $F$ for different values of $R a, \sigma$ and $\psi$ in Figures 16-18. It is observed from Figure 16 that for fixed values of $F$, the flow rate $W$ decreases with an increase in Rayleigh number $R a$. Figure 17 reveals that flow rate $W$ increases with an increase in porosity parameter $\sigma$. On the other hand, it is seen from Figure 18 that for fixed values of $F, W$ decreases with an decrease in $\psi$.

\section{Conclusions}

The fully developed free convection flow of a radiating gas between two vertical thermally conducting walls embedded in porous medium has been studied. The effects of the permeability of the porous medium and the influences of radiation parameter and thermal wall conductances on velocity and temperature fields are investigated and analyzed with the help of their graphical representa-

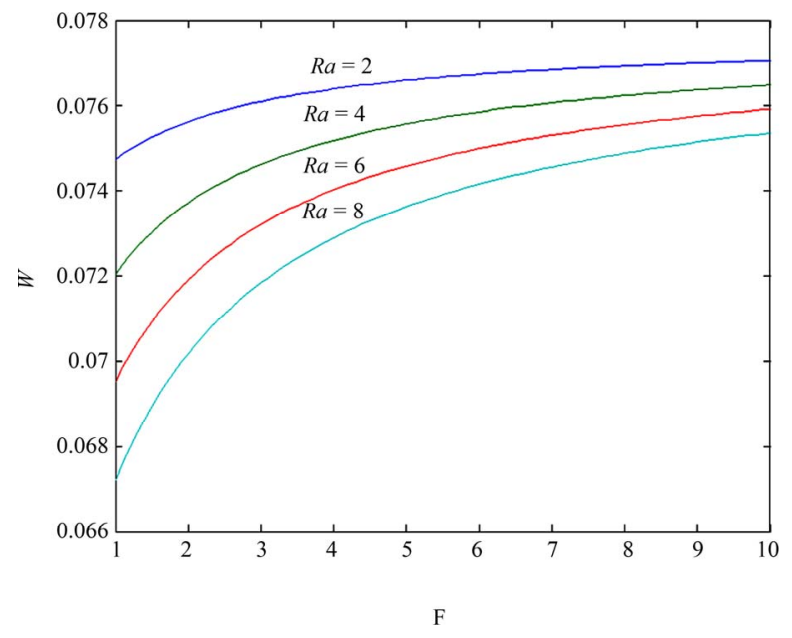

Figure 16. Variations of $W$ against $F$ for $\sigma=0.1$ and $\psi=0.5$.

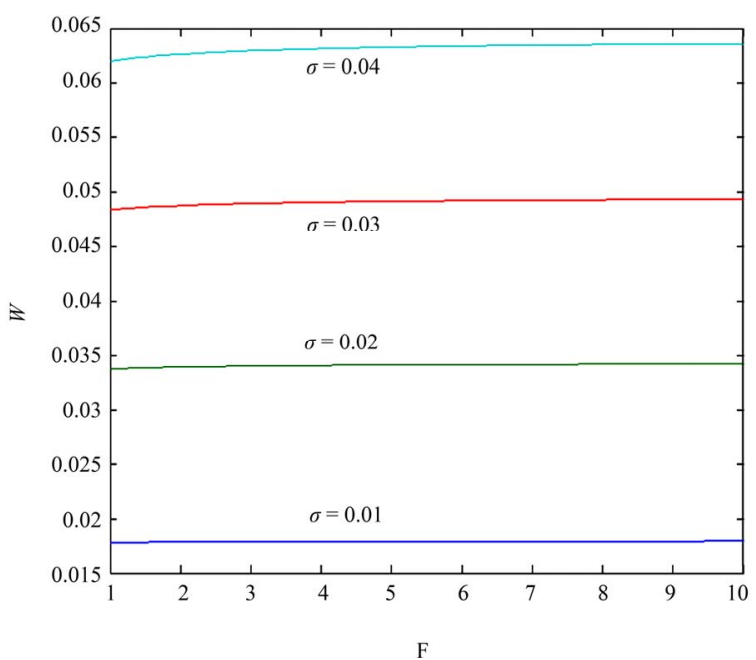

Figure 17. Variations of $W$ against $F$ for $\psi=0.5$ and $R a=2$.

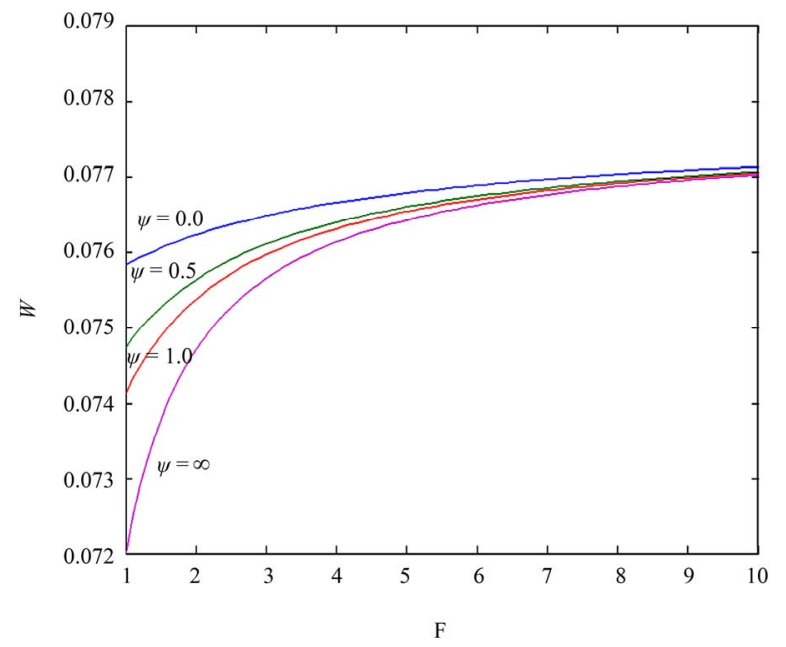

Figure 18. Variations of $W$ against $F$ for $\sigma=0.1$ and $R a=2$.

tions. It is observed that the fluid velocity $u_{1}(\eta)$ increases and the temperature distribution $\theta(\eta)$ decreases with an increase in the radiation parameter $F$. It is also observed that both the fluid velocity and temperature in the flow field increase with an increase in the porosity parameter $\sigma$. It is found that the fluid velocity decreases while the temperature increases with an increase in the thermal conductance of the walls $\psi$. Further, it is found that radiation causes to decrease the rate of heat transfer to the fluid thereby reducing the effect of natural convection. The rate of flow increases with an increase in either $F$ or Rayleigh number $R a$.

\section{References}

[1] D.A. Nield and A.Bejan, "Convection in Porous Media," Springer, Berlin, Heidelberg, New York, 1971. 
[2] E. M. Sparrrow and R. D. Cess, "Radiation Heat Transfer," Hemispherer Publication Corp., Washington DC, 1978.

[3] L. C. Burmeister, “Convective Heat Transfer,” Wiley, New York, 1983.

[4] A. Bejan, “Convection Heat Transfer,” Wiley, New York, 1994.

[5] M. Kavinay, "Principles of Heat Transfer in porous Media,” Springer-Verlay, New York, 1995.

[6] K. Vafai, "Handbook of Porous Media," 2nd Edition, Taylor and Francis, New York, 2005. doi:10.1201/9780415876384

[7] A. Raptis, "Radiation and Free Convection Flow through a Porous Medium," International Communications in Heat and Mass Transfer, Vol. 25, No. 2, 1998, pp. 289295. doi:10.1016/S0735-1933(98)00016-5

[8] A. Raptis, "Radiation and Flow through a Porous Medium,” Journal of Porous Media, Vol. 4, No. 3, 2001, pp. 271-273.

[9] D. Hall, G. C. Vliet and T. L. Bergman, "Natural Convection Cooling of Vertical Rectangular Channels in Air Considering Radiation and Wall Conduction,” Journlaof Electronic Packaging, Vol. 121, No. 2, 1999, pp. 75-84. doi:10.1115/1.2792671

[10] M. A. Al-Nimr and O. H. Haddad, "Fully Developed Free Convection in Open-Ended Vertical Channels Partially Filled with Porous Material," Journal of Porous Media, Vol. 2, No. 2, 1999, pp. 179-189.

[11] A. A. Mohammadein and M. F. El-Amin, "Thermal Dispersion-Radiation Effects on Non-Darcy Natural Convection in a Fluid Saturated Porous Medium," Transport in Porous Medium, Vol. 40, No. 2, 2000, pp. 153-163. doi:10.1023/A:1006654309980

[12] S. H. Kim, N. K. Anand and W. Aung, "Effect of Wall Conduction on Free Convection between Asymmetrically Heated Vertical Plates, Uniform Wall Heat Flux," International Communications in Heat and Mass Transfer,
Vol. 33, No. 5, 1990, pp. 1013-1023. doi:10.1016/0017-9310(90)90082-6

[13] R. Greif, I. S. Habib and J. C. Lin, "Laminar Convection of a Radiating Gas in a Vertical Channel," Journal of Fluid Mechanics, Vol. 46, No. 3, 1971, pp. 513-520. doi:10.1017/S0022112071000673

[14] C. P. Yu and K. K. Yang, "Effect of Wall Conductances on Convective Magnetohydrodynamic Channel Flow,” Applied Scietific Research, Vol. 20, No. 1, 1969, pp. 16-23. doi:10.1007/BF00382379

[15] N. Datta and R. N Jana, "Effect of Wall Conductances on Hydromagnetic Convection of a Radiation Gas in a Vertical Channel," International Communications in Heat and Mass Transfer, Vol. 19, No. 9, 1974, pp. 1015-1019. doi:10.1016/0017-9310(76)90184-8

[16] P. S. Gupta and A. S. Gupta, "Radiation Effect on Hydromagnetic Convection in a Vertical Channel," International Communications in Heat and Mass Transfer, Vol. 17, No. 12, 1973, pp. 1437-1442. doi:10.1016/0017-9310(74)90053-2

[17] O. D. Makinde and P. Y. Mhone, "Heat Transfer to MHD Oscillatory Flow in a Channel Filled with Porous Medium," Romanian Journal of Physics, Vol. 50, No. 9-10, 2005, pp. 931-938.

[18] M. Narahari, "Effects of Thermal Radiation and Free Convection Currents on the Unsteady Couette Flow between Two Vertical Parallel Plates with Constant Heat Flux at One Boundary," WSEAS Transactions on Heat and Mass Transfer, Vol. 1, No. 5, 2010, pp. 21-30.

[19] A. C. Cogley, W. C. Vincenti and S. E. Gilles, "Differential Approximation for Radiative Transfer in a Non-Grey Gas near Equilibrium,” AIAA Journal, Vol. 6, No. 3, 1968, pp. 551-553. doi: $10.2514 / 3.4538$

[20] C. L. Tien, “Thermal Radiation Properties of Gases,” Advances in Heat Transfer, Vol. 5, 1968, pp. 253-324. 